\title{
Target Localization and Circumnavigation by a Non-holonomic Robot
}

\author{
Mohammad Deghat, Edwin Davis, Tianlong See, Iman Shames, Brian D. O. Anderson and Changbin Yu
}

\begin{abstract}
This paper addresses a surveillance problem in which the goal is to achieve a circular motion around a target by a non-holonomic agent. The agent only knows its own position with respect to its initial frame, and the bearing angle of the target in that frame. It is assumed that the position of the target is unknown. An estimator and a controller are proposed to estimate the position of the target and make the agent move on a circular trajectory with a desired radius around it. The performance of the proposed algorithm is verified both through simulations and experiments. Robustness is also established in the face of noise and target motion.
\end{abstract}

\section{INTRODUCTION}

One of the primary applications of unmanned aerial vehicles (UAVs) is in surveillance missions where the UAVs want to monitor and assess an unknown target. A typical way for assessing a target and gathering information about it is to circle around it at a prescribed distance. This problem has recently been studied in the literature, for instance see [1]-[6]. Different control algorithms have been proposed for circumnavigating a target by a single or a group of agents. In order to simplify the design of the control law, most of the previous approaches used a single-integrator holonomic point agent model. In this paper, however, we use the nonholonomic system dynamics that is a more realistic model of a mobile robot than the earlier models considered in the literature. Additionally, we consider that the forward speed of the agent is constant. This assumption along with the nonholonomic dynamics of the agent results in a more accurate model for the motion of low-cost UAVs. Again, many of the current results assume that the position of the target is known and try to find a control algorithm for a single or a group of agents to perform the circumnavigation task. If however the position of the target is initially unknown (as in the case considered in this paper), the agent(s) needs an estimator to localize the target as well as a controller to be able to circumnavigate the target. In this paper we only use the bearing angle of the target and the position of the agent with respect to its initial frame to estimate the target position and control the agent. The advantage of using bearing measurement instead of distance measurement (and also relative position) is that bearing measurement techniques are usually

Mohammad Deghat and Brian D. O. Anderson are with National ICT Australia and Research School of Engineering, The Australian National University, Canberra ACT 0200 .

Email: \{Mohammad.Deghat, Brian.Anderson\}@anu.edu.au

Edwin Davis, Tianlong See and Changbin Yu are with Research School of Engineering, The Australian National University, Canberra ACT 0200.

Email: \{u4669521,u4488286,Brad.Yu\}@anu.edu.au

Iman Shames is with ACCESS Linnaeus Centre, Electrical Engineering, Royal Institute of Technology (KTH). Email: imansh@kth.se

This work is supported by NICTA, which is funded by the Australian Government as represented by the Department of Broadband, Communications and the Digital Economy and the Australian Research Council through the ICT Centre of Excellence program and by ARC grant DP-0877562 and DP-110100538 passive methods, but distance measurement techniques are usually active methods.

In this paper, we propose an estimator and a controller to make a non-holonomic robot move toward and then on a circular trajectory around a target with only the knowledge of its own position and the bearing angle of the target (in its own initial coordinate frame). The estimator we use here is similar to the one in [2], however the stability proof is different because the control law we use here is different. The control law is also a modification of the controller in [4]. The main contributions of this paper are as follows. First, in [4], the non-holonomic robot needs to know the relative position of the target (both distance and bearing), but in this paper, the agent only needs to know the bearing angle. Second, experimental verification of the proposed algorithm is provided using a four-wheeled robot named Wifibot. Third, collision avoidance between the agent and the target is guaranteed and it is shown that under the proposed estimator and controller, the closed loop system is robust against small perturbations due to disturbances or measurement noises.

The rest of this paper is structured as follows. In section II the localization and circumnavigation problem is formally stated. The proposed algorithm is provided in section III. Section IV and V contain simulation and experimental results, respectively. Conclusions and future directions are presented in section VI.

\section{PROBLEM STATEMENT}

Consider a non-holonomic agent moving with a constant forward speed with known trajectory $\boldsymbol{p}_{A}(s)=$ $\left[x_{a}(s), y_{a}(s)\right]^{\top} \in \mathbb{R}^{2}$ for $s \leq t$ at time $t$ and with knowledge of bearing angle of the target $\Gamma(s) \in[0,2 \pi)$ for $s \leq t$. Suppose there is a target with unknown position $\boldsymbol{p}_{T}=\left[x_{t}, y_{t}\right]^{\top} \in \mathbb{R}^{2}$. The coordinate frame corresponds to that of the initial position of the agent. Let $\hat{\boldsymbol{p}}_{T}(t)=$ $\left[\hat{x}_{t}(t), \hat{y}_{t}(t)\right]^{\top} \in \mathbb{R}^{2}$ be the estimated position of the target at time $t, \rho_{d}$ be the desired radius of the circle around the target on which the agent should seek to travel, $\rho(t)$ be the distance between the agent and the target and $\hat{\rho}(t)$ be the distance between the agent and the target estimate at time $t$. Assume that $\rho(0)$ and $\hat{\rho}(0)$ are finite. Also let $\theta(t) \in[0,2 \pi)$ be the heading angle of the agent, $\varphi(t)$ be a unit vector on the line passing through the agent and the target, that is

$$
\boldsymbol{\varphi}(t)=\frac{\boldsymbol{p}_{T}-\boldsymbol{p}_{A}(t)}{\left\|\boldsymbol{p}_{T}-\boldsymbol{p}_{A}(t)\right\|}=\frac{\boldsymbol{p}_{T}-\boldsymbol{p}_{A}(t)}{\rho(t)}
$$

and $\bar{\varphi}(t) \in \mathbb{R}^{2}$ be the unit vector perpendicular to $\varphi(t)$ obtained by $\pi / 2$ clockwise rotation of $\varphi(t)$. The case $s=t$ is depicted in Fig. 1. The symbols $\gamma(t), \gamma_{u}(t)$ and $\boldsymbol{U}$ in Fig. 1 are explained later in the paper. 
Let the estimation error be defined as

$$
\tilde{\boldsymbol{p}}_{T}(t)=\hat{\boldsymbol{p}}_{T}(t)-\boldsymbol{p}_{T}
$$

Knowing $\rho_{d}$, the agent position $\boldsymbol{p}_{A}(s)$ and the unit vector $\varphi(s)$ that shows the bearing angle of the target for $s \leq t$,

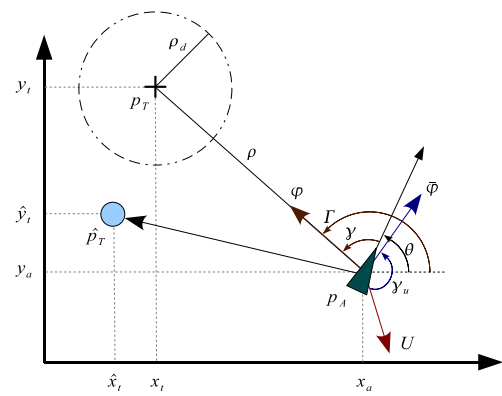

Fig. 1. The agent (triangle), the target (cross) and the estimated position of the target (circle) at time $t$.

entially fast, and also $\rho(t)$ goes to $\rho_{d}$ as time goes to infinity.

We assume that the target is stationary. It can also be shown that if the target experiences slow and possibly persistent movement or if the measurements are noisy, the estimation and control error go to a neighborhood of zero. This is because the exponentially stable systems are robust against system uncertainty and measurement noise [7]. The size of this neighborhood depends on the target speed. In this paper we do not go to the mathematical details of this case and will only provide some simulations in Section IV.

Before proposing the estimator and control law, let us describe the kinematic model of a non-holonomic mobile robot. Consider the planar unicycle model [8]

$$
\begin{aligned}
& \dot{x}_{a}(t)=v \cos \theta(t) \\
& \dot{y}_{a}(t)=v \sin \theta(t) \\
& \dot{\theta}(t)=\omega(t)
\end{aligned}
$$

where $\omega(t) \in \mathbb{R}$ is the agent's angular speed ${ }^{1}$ and $v \in \mathbb{R}$ is its forward speed which is assumed to be constant. This model also describes the model of a fixed-wing UAV flying at a constant altitude. Let $\gamma(t) \in[0,2 \pi)$ be the angular difference between the heading of the agent and the target at time $t$ as shown in Fig. 1. Then for $\rho(t) \neq 0$ the kinematic model of the non-holonomic agent in (3) can be written, after straightforward calculations, as

$$
\begin{aligned}
& \dot{\rho}(t)=-v \cos \gamma(t) \\
& \dot{\gamma}(t)=\frac{v}{\rho(t)} \sin \gamma(t)-\omega(t)
\end{aligned}
$$

\section{PROPOSED ALGORITHM}

The aim of this section is to propose an estimator that estimates the position of the target, and a controller that forces the agent to move toward and then on a desired circular trajectory around the target. We first introduce the estimator and the controller and then prove that they are stable. It is usually desirable, if not essential, to apply estimators and controllers that do not use explicit derivatives of

\footnotetext{
${ }^{1}$ For practical references, there is an upper bound on $\omega(t)$ which should be respected.
}

measurements since differentiation amplifies high-frequency noise. An advantage of the proposed algorithm is that it avoids such differentiations. The estimator we use here has previously been proposed in [2], but the stability proof is different due to the difference in the control law we use in this paper. The estimator can be defined as

$$
\dot{\hat{\boldsymbol{p}}}_{T}(t)=k_{\text {est }}\left(\boldsymbol{I}-\boldsymbol{\varphi}(t) \boldsymbol{\varphi}^{\top}(t)\right)\left(\boldsymbol{p}_{A}(t)-\hat{\boldsymbol{p}}_{T}(t)\right)
$$

where $k_{e s t} \in \mathbb{R}$ is a positive constant, $\boldsymbol{I} \in \mathbb{R}^{2 \times 2}$ is the identity matrix and $\varphi(t) \varphi^{\top}(t)$ is a projection matrix onto the vector $\varphi(t)$. It should be noted that the measurement of the bearing angle to the target when $\rho(t)=0$ is not well defined. Moreover, $\varphi(t)$ is not defined for this case as well. Hence, it is desirable that $\rho(t) \neq 0$ for all $t>0$. It is shown in Appendix I that $\rho(t) \forall t>0$ will never reach zero if $\rho(0) \neq 0$ when the control law proposed below is employed.

Considering (1), (2) and (5), the estimation error dynamics can be written as

$$
\begin{aligned}
\dot{\tilde{\boldsymbol{p}}}_{T}(t) & =k_{\text {est }}\left(\boldsymbol{I}-\boldsymbol{\varphi}(t) \boldsymbol{\varphi}^{\top}(t)\right)\left(-\boldsymbol{\varphi}(t) \rho(t)-\tilde{\boldsymbol{p}}_{T}(t)\right) \\
& \boldsymbol{\varphi}^{\top} \boldsymbol{\varphi}=1 \\
& =k_{\text {est }}\left(\boldsymbol{I}-\boldsymbol{\varphi}(t) \boldsymbol{\varphi}^{\top}(t)\right) \tilde{\boldsymbol{p}}_{T}(t) .
\end{aligned}
$$

It can be easily shown (see Lemma 2 in [2]) that (6) can be written as

$$
\dot{\tilde{\boldsymbol{p}}}_{T}(t)=-k_{\text {est }}\left(\overline{\boldsymbol{\varphi}}(t) \overline{\boldsymbol{\varphi}}^{\top}(t)\right) \tilde{\boldsymbol{p}}_{T}(t) .
$$

Now, we explain the control law that makes the agent move around the target. The control law we propose here is a modified version of the controller in [4]. We assume that the forward speed of the agent is constant, and the angular speed of rotation is controlled by ${ }^{2}$

$$
\omega(t)= \begin{cases}-\omega_{0}+k g(t) \alpha_{d}(t) & \text { if } \hat{\rho}(t)>0 \\ -\omega_{0} & \text { if } \hat{\rho}(t)=0\end{cases}
$$

where

$$
\begin{gathered}
g(t)=\ln \left(\frac{(c-1) \hat{\rho}(t)+\rho_{0}}{c \rho_{0}}\right) \\
\alpha_{d}(t)= \begin{cases}\gamma(t) & \text { if } 0 \leq \gamma(t) \leq \psi \\
\gamma(t)-2 \pi & \text { if } \psi<\gamma(t)<2 \pi\end{cases}
\end{gathered}
$$

and $\omega_{0}>0, k>0, c>1$ and $\rho_{0}>0$ are constant scalars and $\psi \in\left(\frac{3}{2} \pi, 2 \pi\right)$ is a given constant. [Some discussion occurs later on the choice of these quantities.]

The desired rotational motion of the agent around the target is counter-clockwise. However, the threshold $\psi$ is introduced so that when $\hat{\rho}(0)$ is large (and therefore $g(0)$ in (9) is positive) and $\gamma(0)$ is less than but close to $2 \pi\left(\alpha_{d}(0)\right.$ in (10) is negative), $\omega(0)$ in (8) is negative and the agent starts moving clockwise. Fig. 2 shows two different cases where $\psi$ is approximately $3 \pi / 2$ and $2 \pi$. It can be seen that when $\psi$ is approximately $3 \pi / 2$ (the dashed-dotted black line), the agent avoids extra counter-clockwise circular motion and moves more directly to the desired circle. Without defining $\psi$ (or when $\gamma(0) \leq \psi$ ), the agent initially turns counter-clockwise when $\hat{\rho}(0)$ is large and $\gamma(0)$ is less than but close to $2 \pi$

\footnotetext{
${ }^{2}$ We will establish in Appendix I that $\rho(t)$ never goes to zero for $t>0$ in a finite time if $\rho(0) \neq 0$. However, we do not prove a corresponding condition for $\hat{\rho}(t)$.
} 


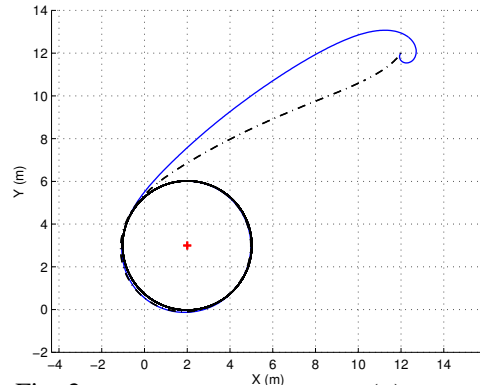

Fig. 2. Agent trajectories when $\gamma(0)$ is less than but close to $2 \pi$ for two different choices of $\psi$. The solid blue line is for the case where $\psi \geq \gamma(0)$ and the dashed-dotted black line is for the case where $\psi<\gamma(0)$.

sented by

$$
\begin{aligned}
& \dot{\rho}(t)=-v \cos \gamma(t) \\
& \dot{\gamma}(t)= \begin{cases}\frac{v}{\rho(t)} \sin \gamma(t)+\omega_{0}-k g(t) \alpha_{d}(t) & \text { if } \hat{\rho}(t)>0 \\
\frac{v}{\rho(t)} \sin \gamma(t)+\omega_{0} & \text { if } \hat{\rho}(t)=0\end{cases}
\end{aligned}
$$

In order to prove the stability of the controller we first establish the stability of the estimator.

Lemma 1: Under the estimator (5) and the control law (8) the estimation error $\tilde{\boldsymbol{p}}_{T}(t)$ described by (2) goes to zero exponentially fast.

In order to prove Lemma 1, we recall the following theorem [9].

Theorem 1: Let $\boldsymbol{V}(\cdot): \mathbb{R}_{+} \rightarrow \mathbb{R}^{n \times r}$ be regulated. Then

$$
\dot{\boldsymbol{x}}=-\boldsymbol{V} \boldsymbol{V}^{\top} \boldsymbol{x}
$$

is exponentially asymptotically stable iff for some positive $\alpha_{1}, \alpha_{2}, T$ and for all $t_{0} \in \mathbb{R}_{+}$

$$
\alpha_{1} \boldsymbol{I} \leq \int_{t_{0}}^{t_{0}+T} \boldsymbol{V}(t) \boldsymbol{V}^{\top}(t) d t \leq \alpha_{2} \boldsymbol{I}
$$

The condition (13) is called a persistence of excitation condition and $\boldsymbol{V}(\cdot)$ is said to be persistently exciting (p.e.) if it satisfies (13). Another interpretation of the p.e. condition (13) in scalar form is that there exist positive $\epsilon_{1}, \epsilon_{2}$ and $T$ such that

$$
\epsilon_{1} \leq \int_{t_{0}}^{t_{0}+T}\left(\boldsymbol{U}^{\top} \boldsymbol{V}(t)\right)^{2} d t \leq \epsilon_{2} \quad \forall t_{0} \in \mathbb{R}_{+}
$$

where $\boldsymbol{U} \in \mathbb{R}^{n}$ can be any constant unit vector [10].

Proof of Lemma 1: Considering (7) and Theorem 1, the signal $\tilde{\boldsymbol{p}}_{T}(t)$ exponentially goes to zero if and only if $\overline{\boldsymbol{\varphi}}(t)$ is persistently exciting. The condition on $\bar{\varphi}(t)$ to be p.e. is that there exist some positive $\epsilon_{1}, \epsilon_{2}$, and $T$, such that

$$
\epsilon_{1} \leq \int_{t_{0}}^{t_{0}+T}\left(\boldsymbol{U}^{\top} \overline{\boldsymbol{\varphi}}(t)\right)^{2} d t \leq \epsilon_{2}
$$

is satisfied for all constant unit length $\boldsymbol{U} \in \mathbb{R}^{2}$ and all $t_{0} \in$ $\mathbb{R}_{+}$. Assume $\gamma_{u}(t)$ is the angle from the unit vector $\boldsymbol{U}$ to the unit vector $\bar{\varphi}(t)$ (as shown in Fig. 1 for a sample $\boldsymbol{U}$ ). Then (15) can be written as

$$
\epsilon_{1} \leq \int_{t_{0}}^{t_{0}+T} \cos ^{2} \gamma_{u}(t) d t \leq \epsilon_{2}
$$

Since $\cos ^{2}(\cdot) \leq 1$, the integral in (16) is always bounded from above and an upper bound for (16) is $\epsilon_{2}=T$. On the other hand, $\cos ^{2}(\cdot) \geq 0 \forall t \geq 0$ and therefore $\epsilon_{1} \geq 0$. We need to show that $\epsilon_{1}>0$.

If we find some constant scalar $\delta_{1}>0$ such that for some positive $T$ and all $t_{0}>0,\left|\dot{\gamma}_{u}(t)\right|>\delta_{1} \forall t_{0}<t<t_{0}+T$ then the proof will be complete and $\bar{\varphi}(t)$ will be persistently exciting. Since $\varphi(t)$ is perpendicular to $\bar{\varphi}(t)$ and the unit vector $\boldsymbol{U}$ is a constant vector, one has

$$
\dot{\gamma}_{u}(t)=\dot{\Gamma}(t)
$$

Then by considering the second equation of (4) and noting that $\Gamma(t)=\gamma(t)+\theta(t) \quad \bmod (2 \pi)$ (See Fig. 1) one obtains that

$$
\dot{\gamma}_{u}(t)=\frac{v}{\rho(t)} \sin \gamma(t)
$$

It can be observed that the only cases where $\left|\dot{\gamma}_{u}(t)\right|$ is not greater than a sufficiently small $\delta_{1}$ are when $\rho(t) \rightarrow \infty$ or when $\sin \gamma(t) \rightarrow 0$. According to Appendix II, $\rho(t)$ is bounded. So $\left|\dot{\gamma}_{u}(t)\right|$ cannot go to zero because of $\rho(t)$. But depending on the initial value of $\gamma(t),\left|\dot{\gamma}_{u}(t)\right|$ might be very small and even zero. We will show that there exist sufficiently small positive scalars $\delta_{2}$ and $\delta_{3}$ such that all trajectories with $\gamma(t):|\sin \gamma(t)|<\delta_{2}$ will ultimately leave this neighborhood of $\sin \gamma(t)=0$ in a finite time $t_{1}$ and will stay outside this neighborhood for a sufficiently large time $t_{2}$. Then by choosing $T=t_{1}+t_{2}$, the proof will be complete.

If $\hat{\rho}(t)=0$ then because of (11), $\dot{\gamma}(t)=\frac{v}{\rho(t)} \sin \gamma(t)+\omega_{0}$. Suppose there exists a positive scalar $\rho_{\text {min }}>0$ such that $\rho(t) \geq \rho_{\min }$ for all $t \geq 0 .^{3}$ Then for a sufficiently small scalar $\delta_{2}>0$, one can find a scalar $\delta_{3}>0$ such that for $|\sin \gamma(t)|<\delta_{2}, \dot{\gamma}(t)>\delta_{3}>0$. In this case, the finite time $t_{1}$ would be at most $2 \delta_{2} / \delta_{3}$. Also, since $\dot{\gamma}(t)$ is bounded, there is a sufficiently large period of time, $t_{2}$, such that $\gamma(t)$ is outside the set $\left\{\gamma(t):|\sin \gamma(t)|<\delta_{2}\right\}$.

If $\hat{\rho}(t)>0$, then according to (11) on has

$$
\dot{\gamma}(t)=\frac{v}{\rho(t)} \sin \gamma(t)+\omega_{0}-k \alpha_{d}(t) \ln \left(\frac{(c-1) \hat{\rho}(t)+\rho_{0}}{c \rho_{0}}\right)
$$

We first consider the case where $0 \leq \gamma(t)<\delta_{2}^{\prime}$ or $2 \pi-\delta_{2}^{\prime}<$ $\gamma(t)<2 \pi$ for a sufficiently small $\delta_{2}^{\prime}>0$. Since $\hat{\rho}(t)$ is bounded (see Appendix II), one can find some $\delta_{3}>0$ such that $\dot{\gamma}(t)>\delta_{3}>0$. In this case, $t_{1}$ would be at most $2 \delta_{2}^{\prime} / \delta_{3}$. Note that the trajectories cannot enter this neighborhood from the boundary $\gamma=\delta_{2}^{\prime}$ because $\dot{\gamma}(t)>\delta_{3}>0$ when $\gamma<\delta_{2}^{\prime}$.

If $\pi-\delta_{2}^{\prime}<\gamma(t)<\pi+\delta_{2}^{\prime}$ then according to the first equation of (11), $\dot{\rho}(t) \approx v$ and therefore the value of $\rho(t)$ gets larger. In this case, the first term on the right hand side of (19) is about zero (because $\gamma(t) \approx \pi$ ) and because of the triangle inequality $\hat{\rho}(t) \geq \rho(t)-\left\|\tilde{\boldsymbol{p}_{T}}(t)\right\| \geq \rho(t)-\left\|\tilde{\boldsymbol{p}_{T}}(0)\right\|, \hat{\rho}(t)$ is lower bounded by an increasing function. So after some time, the last term on the right hand side of (19) becomes larger in magnitude than $\omega_{0}$ and for a sufficiently small positive constant $\delta_{3}$, there exists a finite time $t_{1}$ such that $\dot{\gamma}\left(t_{1}\right)<$

\footnotetext{
${ }^{3}$ Note that such a lower bound for $\rho(t)$ can always be found if $\rho(0) \neq 0$. If $\rho(t)$ is close to $0(\rho(t)$ cannot be exactly zero because of Lemma 5 in Appendix I), then according to the proof of Lemma 5, $\gamma(t)$ converges to $\pi$ very fast and it remains at $\pi$ until the agent is sufficiently far away from the target, i.e., $\rho(t)$ is sufficiently large.
} 
$-\delta_{3}$. Since $\dot{\rho}(t)$ is positive for $\gamma(t) \in(\pi / 2,3 \pi / 2)$, the lower bound of $\hat{\rho}(t)$ increases as long as $\gamma(t) \in(\pi / 2,3 \pi / 2)$.

The last thing to show is that $\gamma(t)$ does not immediately go back to the neighborhood of $\gamma(t):|\sin \gamma(t)|<\delta_{2}$ and there exists a sufficiently large time $t_{2}$ such that $\gamma(t)$ is out of that neighborhood. As explained above, the trajectories cannot enter the neighborhood $0 \leq \gamma(t)<\delta_{2}^{\prime}$ or $2 \pi-\delta_{2}^{\prime}<$ $\gamma(t)<2 \pi$ from the boundary $\gamma=\delta_{2}^{\prime}$ because $\dot{\gamma}(t)>\delta_{3}>0$ when $\gamma(t)<\delta_{2}^{\prime}$. It also takes a finite time for trajectories that leave this neighborhood to enter the neighborhood $\pi-\delta_{2}^{\prime}<$ $\gamma(t)<\pi+\delta_{2}^{\prime}$, because $|\dot{\gamma}(t)|$ in (19) is bounded. Hence, there is a sufficiently large period of time, $t_{2}$, such that $\gamma(t)$ stays in $\left(\delta_{2}^{\prime}, 2 \pi-\delta_{2}^{\prime}\right)$ in that period. It can similarly be shown that the trajectories leaving $\pi-\delta_{2}^{\prime}<\gamma(t)<\pi+\delta_{2}^{\prime}$ do not immediately return back to this neighborhood.

Having established that the estimation error exponentially converges to zero, we should show that the controller also works properly and $\rho(t)$ goes to $\rho_{d}$ as $t \rightarrow \infty$. Since the estimation error exponentially goes to zero, it is reasonable to assume that there exists a time $\tau$ that for all time $t \geq \tau$, $\hat{\boldsymbol{p}}_{T}(t) \approx \boldsymbol{p}_{T}$. So for the rest of this section we assume that $\rho(t)=\hat{\rho}(t)$. Then (8)-(10) can be written as

$$
\begin{gathered}
\omega(t)= \begin{cases}-\omega_{0}+k g(t) \alpha_{d}(t) & \text { if } \rho(t)>0 \\
-\omega_{0} & \text { if } \rho(t)=0\end{cases} \\
g(t)=\ln \left(\frac{(c-1) \rho(t)+\rho_{0}}{c \rho_{0}}\right) \\
\alpha_{d}(t)= \begin{cases}\gamma(t) & \text { if } 0 \leq \gamma(t) \leq \psi \\
\gamma(t)-2 \pi & \text { if } \psi<\gamma(t)<2 \pi\end{cases}
\end{gathered}
$$

In order to prove the stability of the closed loop system, we first show that the closed loop system has only one equilibrium point corresponding to the counter-clockwise rotation of the vehicle around the target.

Proposition 1: If the constants $v>0, k>0, c>1$ and $\rho_{0}>0$ are chosen such that

$$
\min _{\rho}\left(\frac{3 \pi}{2} k g\left(\rho ; c, \rho_{0}\right)+\frac{v}{\rho}\right)>\omega_{0}
$$

then the point $\left(\rho_{d}, \pi / 2\right)$ where $\rho_{d}$ is such that

$$
\frac{v}{\rho_{d}}-k g\left(\rho_{d} ; c, \rho_{0}\right) \cdot \frac{\pi}{2}+\omega_{0}=0
$$

is the only equilibrium of the system (4) and (20)-(22) for $(\rho, \gamma)$ in the set $\mathcal{D}=\mathbb{R}_{+} \times(0,2 \pi)$ where $\mathbb{R}_{+}$denotes the set of positive real numbers and $g\left(\rho_{d} ; c, \rho_{0}\right)$ is the function defined in (21) in which $\rho(t)=\rho_{d}$.

To check the stability of the closed loop system, let us consider the following Lyapunov function, inspired from [4], on $\mathcal{D}=\mathbb{R}_{+} \times(0,2 \pi)$.

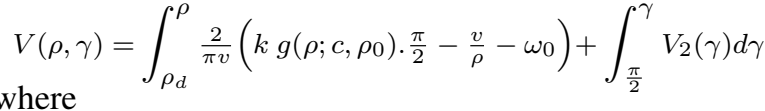

$$
\begin{aligned}
& V_{2}(\gamma)=\left\{\begin{aligned}
-\frac{\cos \gamma}{\gamma} & \text { if } 0<\gamma \leq \psi \\
-\frac{\cos \gamma}{\gamma-2 \pi} & \text { if } \psi<\gamma<2 \pi
\end{aligned}\right.
\end{aligned}
$$

It can be easily checked that $V(\rho, \gamma) \geq 0 \forall(\rho, \gamma) \in \mathcal{D}$. Moreover $V(\rho, \gamma)=0$ only for $\rho=\rho_{d}$ and $\gamma=\pi / 2$, and
$V(\rho, \gamma)$ is radially unbounded on $\mathcal{D}$. Thus $V(\rho, \gamma)$ is a valid Lyapunov function candidate whose derivative is

$$
\dot{V}= \begin{cases}\left(\frac{v}{\rho}\left(\frac{2}{\pi}-\frac{\sin \gamma}{\gamma}\right)+\omega_{0}\left(\frac{2}{\pi}-\frac{1}{\gamma}\right)\right) \cos \gamma & \text { if } 0<\gamma \leq \psi \\ \left.\frac{v}{\rho}\left(\frac{2}{\pi}-\frac{\sin \gamma}{\gamma-2 \pi}\right)+\omega_{0}\left(\frac{2}{\pi}-\frac{1}{\gamma-2 \pi}\right)\right) \cos \gamma & \text { if } \psi<\gamma<2 \pi\end{cases}
$$

It can be observed that $\dot{V}(\rho, \gamma) \leq 0 \forall(\rho, \gamma) \in \overline{\mathcal{D}}=\mathbb{R}_{+} \times$ $\left(0, \frac{3 \pi}{2}\right]$ if $\omega_{0}>0$.

The following lemma shows that any trajectory starting outside $\overline{\mathcal{D}}$ will end up in $\overline{\mathcal{D}}$ in a bounded time which depends on $\gamma(0)$ and $\rho(0)$, and any trajectory in $\overline{\mathcal{D}}$ will stay in $\overline{\mathcal{D}}$. Then we will show that any trajectory starting in $\overline{\mathcal{D}}$ converges to $\rho=\rho_{d}$ and $\gamma=\pi / 2$.

Lemma 2: Let $\mathcal{D}=\mathbb{R}_{+} \times(0,2 \pi), \overline{\mathcal{D}}=\mathbb{R}_{+} \times\left(0, \frac{3 \pi}{2}\right]$ and $\hat{\mathcal{D}}=\mathcal{D} \backslash \overline{\mathcal{D}}$. Then for any trajectory of (4) with initial condition in $\hat{\mathcal{D}} \cup\{(\rho(t), \gamma(t)): \gamma(t)=0\}$, there exists a finite time $\bar{t}$ such that $(\rho(\bar{t}), \gamma(\bar{t})) \in \overline{\mathcal{D}}$. Furthermore, any trajectory in $\overline{\mathcal{D}}$ will stay in $\overline{\mathcal{D}}$.

Proof: We first show that any trajectory starting in $\overline{\mathcal{D}}$ will stay in $\overline{\mathcal{D}}$. To this end, we check all the boundaries of $\overline{\mathcal{D}}$ which are $\gamma=0, \gamma=3 \pi / 2$ and $\rho=0$. According to Appendix I, $\rho(t)$ does not go to zero if $\rho(0) \neq 0$. So, the trajectories in $\overline{\mathcal{D}}$ cannot leave $\overline{\mathcal{D}}$ through $\rho(t)=0$. If for some $0 \leq t_{1}<\infty, \gamma\left(t_{1}\right)=0$ then $\dot{\gamma}\left(t_{1}\right)=\omega_{0}>0$. Also if $\gamma\left(t_{1}\right)=3 \pi / 2$ then according to (4) and (20)-(22), $\dot{\gamma}\left(t_{1}\right)=-\frac{v}{\rho\left(t_{1}\right)}+\omega_{0}-\frac{3 \pi}{2} k g\left(t_{1}\right)$ and according to (23), $\dot{\gamma}\left(t_{1}\right)<0$. So the trajectories in $\overline{\mathcal{D}}$ cannot leave $\overline{\mathcal{D}}$.

The next step is to show that the trajectories in $\hat{\mathcal{D}} \cup$ $\{(\rho(t), \gamma(t)): \gamma(t)=0\}$ will go to $\overline{\mathcal{D}}$ in finite time. If $\gamma(t)=0$, then according to (4) and (20)-(22), $\dot{\gamma}(t)=\omega_{0}>$ 0 . So, the trajectory whose $\gamma(t)=0$ will go to $\overline{\mathcal{D}}$. Let $\hat{\mathcal{D}}_{1}=\mathbb{R}_{+} \times\left(\frac{3 \pi}{2}, \beta\right)$ and $\hat{\mathcal{D}}_{2}=\mathbb{R}_{+} \times(\beta, 2 \pi)$ where $\beta$ is such that $\dot{\gamma}<-\zeta<0$ for any $(\rho, \gamma) \in \hat{\mathcal{D}}_{1}$ and for some $\zeta>0$. Note that such $\zeta$ can always be found because of (23). Now consider any initial condition in $\hat{\mathcal{D}}_{2}$. Since $\dot{\rho} \leq-v \cos \zeta<0$ and there cannot exist trajectories such that $\rho(t)=0$, the longest period of time that any trajectory can stay in $\hat{\mathcal{D}}_{2}$ is $\bar{t}_{1} \leq \frac{\rho(0)}{v \cos \zeta}$. This is the time taken for $\rho(t)$ to get very close to zero. After that, according to (4) and (20)-(22), $\dot{\gamma}(t)$ will go to $-\infty$ and the trajectory will go to $\hat{\mathcal{D}}_{1}$. Hence for any trajectory starting in $\hat{\mathcal{D}}_{2}$, one can always find a time $\bar{t}_{1} \leq \frac{\rho(0)}{v \cos \zeta}$ such that $\left(\rho\left(\bar{t}_{1}\right), \gamma\left(\bar{t}_{1}\right)\right) \in \hat{\mathcal{D}}_{1}$. Now choose any initial condition $(\rho(0), \gamma(0)) \in \hat{\mathcal{D}}_{1}$. Since $\dot{\gamma}<-\zeta$, one can always find a time $\bar{t}_{2} \leq \frac{\gamma(0)-3 \pi / 2}{\zeta}$ such that $\gamma\left(\bar{t}_{2}\right)=3 \pi / 2$. Therefore for any initial condition in $\hat{\mathcal{D}}$ there exists a finite time $\bar{t} \leq \bar{t}_{1}+\bar{t}_{2}$ such that $(\rho(\bar{t}), \gamma(\bar{t})) \in \overline{\mathcal{D}}$.

Considering (4), (20)-(22) and Lemma 2, the closed loop system in $\overline{\mathcal{D}}$ boils down to

$$
\left[\begin{array}{l}
\dot{\rho}(t) \\
\dot{\gamma}(t)
\end{array}\right]=\boldsymbol{f}(\rho(t), \gamma(t))=\left[\begin{array}{c}
-v \cos \gamma(t) \\
\frac{v}{\rho} \sin \gamma(t)-k g(t) \gamma(t)+\omega_{0}
\end{array}\right] .
$$

We need to show the stability of the above system. To this end, we first show that (28) has a unique equilibrium point corresponding to the counter-clockwise rotation of the vehicle around the target. If and only if $\gamma=\frac{\pi}{2}$ or $\gamma=\frac{3 \pi}{2}$ $\bmod (2 \pi)$, then $\dot{\rho}=0$. We want the system to have only one 
equilibrium at $\gamma=\frac{\pi}{2}$. So we should set $v>0, k>0, c>1$ and $\rho_{0}>0$ such that

and

$$
\frac{v}{\rho_{d}}-k g\left(\rho_{d} ; c, \rho_{0}\right) \cdot \frac{\pi}{2}+\omega_{0}=0
$$

$$
-\frac{v}{\rho_{d}}-k g\left(\rho_{d} ; c, \rho_{0}\right) \cdot \frac{3 \pi}{2}+\omega_{0} \neq 0
$$

Let $v, k, c$ and $\rho_{0}$ be chosen such that

$$
\min _{\rho}\left(\frac{3 \pi}{2} k g\left(\rho ; c, \rho_{0}\right)+\frac{v}{\rho}\right)>\omega_{0}
$$

then it is easy to show that the only equilibrium of (28) is $\gamma_{e}=\pi / 2$ and $\rho=\rho_{d}$ where $\rho_{d}$ is the only solution of (29). So, a proper choice of $v, k, c$ and $\rho_{0}$ that satisfies (31) guaranties that $\dot{\gamma}<0$ for $\gamma=3 \pi / 2$.

Lemma 3: Consider any initial condition $(\rho(0), \gamma(0)) \in \overline{\mathcal{D}}$ and the Lyapunov function in (25). Define $\eta=V(\rho(0), \gamma(0))$ and $\mathcal{S}_{\eta}=\{(\rho, \gamma) \in \overline{\mathcal{D}}: V(\rho, \gamma) \leq \eta\}$. Then $\mathcal{S}_{\eta}$ is a compact and positively invariant set with respect to (28).

Proof: Since $V(\rho, \gamma)$ is radially unbounded, the set $\mathcal{S}_{\eta}$ is compact. The boundaries of this compact set are $\partial \mathcal{S}_{\eta}=\{(\rho, \gamma) \in \overline{\mathcal{D}}:(V(\rho, \gamma)=\eta \wedge \gamma<3 \pi / 2) \vee$ $(V(\rho, \gamma) \leq \eta \wedge \gamma=3 \pi / 2)\}$. Consider $V(\rho, \gamma) \in \partial \mathcal{S}_{\eta}$ such that $V(\rho, \gamma)=\eta$ and $\gamma<3 \pi / 2$. Since $\dot{V}(\rho, \gamma) \leq 0$, the trajectories in $\mathcal{S}_{\eta}$ do not exit $\mathcal{S}_{\eta}$ through this boundary. Consider now any $(\rho, \gamma)$ such that $\gamma=3 \pi / 2$. Because of (23), $\dot{\gamma}<0$. Since the right hand side of (28) is continuous and Lipschitz on $\mathcal{S}_{\eta}$ and the set $\mathcal{S}_{\eta}$ contains the equilibrium $\left(\rho_{d}, \pi / 2\right)$, the unique solution of (28) will not leave $\mathcal{S}_{\eta}$ for $t \geq 0$.

Lemma 4: Let $\mathcal{S}_{\eta}$ be as defined in Lemma 3. Then the equilibrium point $\left(\rho_{d}, \gamma_{e}=\frac{\pi}{2}\right)$ is a locally exponentially stable equilibrium for the system (28) and all trajectories starting in $\mathcal{S}_{\eta}$ will end up in that equilibrium.

Proof: The linearized equation of (28) around its equilibrium $\left(\rho_{d}, \frac{\pi}{2}\right)$ is

where

$$
\left[\begin{array}{c}
\dot{\rho}(t) \\
\dot{\gamma}(t)
\end{array}\right]=\boldsymbol{A}\left[\begin{array}{l}
\rho(t) \\
\gamma(t)
\end{array}\right]
$$

$$
\boldsymbol{A}=\left[\begin{array}{cc}
0 & v \\
-\frac{v}{\rho_{d}^{2}}-\frac{k \pi(c-1)}{2\left((c-1) \rho_{d}+\rho_{0}\right)} & -k g\left(\rho_{d} ; c, \rho_{0}\right)
\end{array}\right]
$$

and the eigenvalues of $\boldsymbol{A}$ are

$$
-\frac{k g\left(\rho_{d} ; c, \rho_{0}\right)}{2} \pm \frac{1}{2} \sqrt{k^{2} g^{2}\left(\rho_{d} ; c, \rho_{0}\right)-4 v\left(\frac{v}{\rho_{e}^{2}}+\frac{k \pi(c-1)}{2\left((c-1) \rho_{d}+\rho_{0}\right)}\right)}
$$

Since $\boldsymbol{A}$ is Hurwitz, $\boldsymbol{f}(\rho(t), \gamma(t))$ is continuously differentiable in $\mathcal{S}_{\eta}$ and the jacobian of $\boldsymbol{f}(\rho, \gamma)$ is bounded and Liptschitz on $\mathcal{S}_{\eta}$, then $\left(\rho_{d}, \frac{\pi}{2}\right)$ is a locally exponentially stable equilibrium point for (28) (Theorem 4.15 of [7]).

Recall that $\mathcal{S}_{\eta}$ is a positively invariant set, $V(\rho, \gamma)>0$ in $\mathcal{S}_{\eta}$ except for $\left(\rho_{d}, \pi / 2\right)$ where $V\left(\rho_{d}, \pi / 2\right)=0$, and $\dot{V}(\rho, \gamma) \leq 0$. Then by applying LaSalle's invariant principle one can conclude that the trajectories starting in $\mathcal{S}_{\eta}$ converge asymptotically ${ }^{4}$ to $\left(\rho_{d}, \pi / 2\right)$.

\footnotetext{
${ }^{4}$ Note that this system is both asymptotically stable and locally exponentially stable. If a system is exponentially stable, then it is robust against system uncertainties [7]. So if the target moves slowly, the estimation error goes to a neighborhood of zero and $\rho(t)$ goes to a neighborhood of $\rho_{d}$
}

\section{SIMULATIONS}

Simulation results using Matlab are presented in this section. We assume that $v=3, k_{\text {est }}=2, \boldsymbol{p}_{T}=[2,3]^{\top}$, $\boldsymbol{p}_{A}(0)=[12,12]^{\top}, \hat{\boldsymbol{p}}_{T}(0)=[4,5]^{\top}$, and the constants in (8) and (9) are $\omega_{0}=0.97, k=1, c=2, \rho_{0}=0.5$ and therefore the desired radius of the circle is $\rho_{d}=3$. Also the initial heading of the agent is $2 \pi / 3$. The trajectory of the agent is shown in Fig. 3(a). The estimation error and the distance between the agent and the target are shown in Fig. 3(b). It can be seen that the distance between the agent and the target goes to the desired distance $\rho_{d}=3$ and the estimation error also goes to zero exponentially fast.

Then, we consider the case where the target moves slowly and $\boldsymbol{p}_{T}(t)=[2+.025 t, 3+\sin (.025 t)+.025 t]^{\top}$. The trajectories of the center of rotation of the agent and the target are shown in Fig. 3(c). The estimation and the tracking error are also shown in Fig. 3(d). It can be seen that these errors go to neighborhoods of zero. Note that the sizes of these neighborhoods depend on the speed of the target.

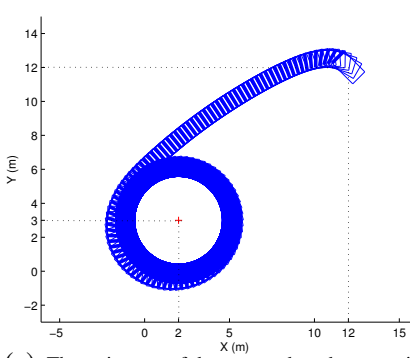

(a) The trajectory of the agent when the target is stationary.

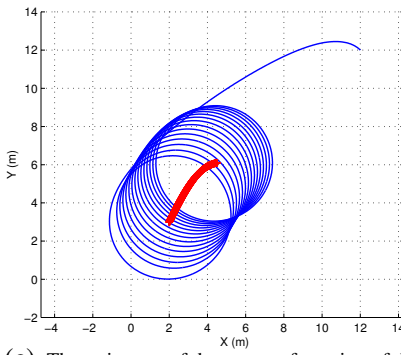

(c) The trajectory of the center of rotation of tuc (d) The distance between the agent and the target agent (blue) and the target (red) when the target (dashed-dotted line) and the estimation error (solid moves slowly.
Fig. 3. Simulation results

\section{EXPERIMENTS}

\section{A. Experimental testbed}

The implementation of the controller was done on a Wifibot (http://www.wifibot.com) shown in Fig. 4. This mobile device has four wheels each independently driven by a $12 \mathrm{~V}$ DC motor with 51:1 gearbox. Two of the motors also feature encoders for odometry measurement. An onboard computer with a $1.6 \mathrm{GHz}$ Atom processor and Gentoo Linux control the operation of the robot. Communication with the robot is achieved over Wifi using Player (http://playerstage.sourceforge.net). The Player runs on the Wifibot and handles communication with the hardware and client code. The Player client code is written in $\mathrm{C} / \mathrm{C}++$ and connects to the server via a network connection to provide 
control of the robot. This structure allows the server to abstract hardware interfaces into higher level functions, such

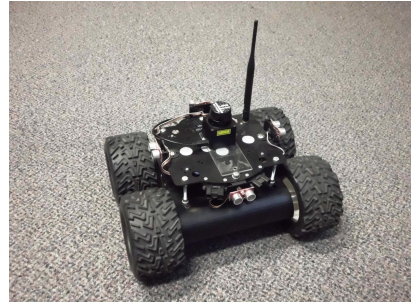

Fig. 4. The Wifibot with Hokuyo URG-04LX-UG01 scanner used in the experiments.

as forward speed, for clearer development of control code. So by using Player, we only need to specify, e.g., forward velocities in $\mathrm{m} / \mathrm{s}$ instead of motor speed. A Hokuyo URG-04LX-UG01 scanning laser range finder is attached to the Wifibot. It has a range of $20 \mathrm{~mm}$ to $5600 \mathrm{~mm}$ at an accuracy of $3 \%$ above $1 \mathrm{~m}$ and an angular range of $240^{\circ}$ at a resolution of $0.352^{\circ}$. This sensor is used for detection of the target and determination of bearing. The position of the robot is also measured using the encoders installed on it.

\section{B. Results}

Since the range of the laser scanner is up to $5.6 \mathrm{~m}$, we could not start far from the target. The agent was initially at $p_{A}(0)=[0,0]^{\top}$, and the target was a cylinder located at

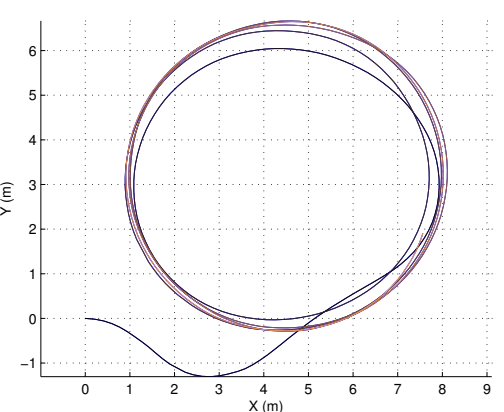

Fig. 5. The trajectory of the robot in the experiment. increases, the trajectory becomes lighter. It can be seen that in the first two rounds, the agent is not completely on the circle, but after that, it converges to the desired circle.

\section{CONCLUSIONS AND FUTURE WORK}

In this paper we proposed an estimator and a controller to make a non-holonomic agent circumnavigate a target with unknown position. It is shown that both the control and estimation error converge to zero when the target is stationary. The cases where the target is not stationary and the measurements are noisy are also investigated in simulation and experiment. Future directions of research include investigating the case where more than one agent or more that one target are present. In this case, collision avoidance techniques should be considered to avoid agents colliding with each other. It should also be guaranteed that the distances between the agents are not larger than their communication range.

\section{APPENDIX I}

Lemma 5: Let $\rho(0) \neq 0$ and consider (4) and (8)-(10). Then $\rho(t) \forall t>0$ will never reach zero.

Proof: $\quad$ Let $\mathcal{R}=\mathbb{R}_{+} \times\left(\frac{\pi}{2}, \frac{3}{2} \pi\right)$. Then for any $(\rho(t), \gamma(t)) \in \mathcal{R}$ one has $\dot{\rho}(t)>0$ and therefore $\rho(t)$ cannot reach zero on this region because $\rho(t)$ is increasing. Suppose that $\rho(0) \neq 0$. Then for $\gamma \in\left(0, \frac{\pi}{2}\right)$, if $\rho(0) \rightarrow 0$, then because of (4), $\dot{\gamma}(t) \rightarrow+\infty$. Similarly, for $\gamma \in\left(\frac{3 \pi}{2}, 2 \pi\right)$, if $\rho(0) \rightarrow 0$ then $\dot{\gamma}(t) \rightarrow-\infty$. So, the trajectories whose $\rho(0)$ is close to zero and $\gamma(0) \in\left(0, \frac{\pi}{2}\right) \cup\left(\frac{3}{2} \pi, 2 \pi\right)$ will go to $\mathcal{R}$ very fast and will stay in $\mathcal{R}$ until the value of $\rho(t)$ is large enough. Also, if $\gamma(0)=0$ then according to (4) one has $\dot{\gamma}(0)=\omega_{0}$. Indeed, if $\rho(0) \rightarrow 0$, then $\gamma(t)$ goes to $\pi$ and the robot moves away from the target until it is sufficiently far away from the target ${ }^{5}$.

\section{APPENDIX II}

Lemma 6: Consider (4), (7) and (8)-(10). If $\rho(0)$ and $\hat{\rho}(0)$ are bounded then $\rho(t)$ and $\hat{\rho}(t)$ are bounded for all $t>0$.

Proof: We first show that the estimation error in (2) is bounded. By choosing $V_{1}=\frac{1}{2} \tilde{\boldsymbol{p}}_{T}^{\top} \tilde{\boldsymbol{p}}_{T}$ and by considering (7), it can be seen that $\dot{V}_{1}=-k_{\text {est }} \tilde{\boldsymbol{p}}_{T}^{\top} \overline{\boldsymbol{\varphi}} \bar{\varphi}^{\top} \tilde{\boldsymbol{p}}_{T}=$ $-k_{e s t}\left\|\bar{\varphi}^{\top} \tilde{\boldsymbol{p}}_{T}\right\|^{2}$ is negative semi-definite and (7) is uniformly stable. Therefore $\tilde{\boldsymbol{p}}_{T}(t)$ is bounded and $\left\|\tilde{\boldsymbol{p}}_{T}(t)\right\| \leq$ $\left\|\tilde{\boldsymbol{p}}_{T}(0)\right\|$. Using the triangle inequality, we have

$$
|\rho(t)-\hat{\rho}(t)| \leq\left\|\tilde{\boldsymbol{p}}_{T}(t)\right\| \leq\left\|\tilde{\boldsymbol{p}}_{T}(0)\right\|
$$

So if we show that $\rho(t)$ is bounded, then according to the above equation we can conclude that $\hat{\rho}(t)$ is also bounded. We now assume that $\rho(t)$ is much larger than $\left\|\tilde{\boldsymbol{p}}_{T}(0)\right\|$. So according to (35), $\hat{\rho}(t)$ is also very large. Then $g(t)$ in (9) will go to infinity and according to (10) and (11), $\frac{\dot{\gamma}(t)}{\gamma} \approx-k g(t)$ when $\gamma(t) \in[0, \psi]\left(\frac{\dot{\gamma}(t)}{\gamma-2 \pi} \approx-k g(t)\right.$ when $\left.\gamma(t) \in(\psi, 2 \pi)\right)$. So $\gamma(t)$ will go to zero very fast and according to the first equation of (4), $\dot{\rho}(t)=-v$ after that and therefore $\rho(t)$ will decrease. So $\rho(t)$ cannot go to infinity and therefore both $\rho(t)$ and $\hat{\rho}(t)$ are bounded.

\section{REFERENCES}

[1] I. Shames, S. Dasgupta, B. Fidan, and B. D. O. Anderson, "Circumnavigation using distance measurements under slow drift," IEEE Transactions on Automatic Control, vol. 57, no. 4, pp. 889-903, 2012.

[2] M. Deghat, I. Shames, B. D. O. Anderson, and C. Yu, "Target localization and circumnavigation using bearing measurements in 2D." 2010 49th IEEE Conference on Decision and Control (CDC), 2010, pp. $334-339$.

[3] J. A. Marshall, M. E. Broucke, and B. A. Francis, "Pursuit formations of unicycles," Automatica, vol. 42, no. 1, pp. 3-12, 2006.

[4] N. Ceccarelli, M. Di Marco, A. Garulli, and A. Giannitrapani, "Collective circular motion of multi-vehicle systems," Automatica, vol. 44, no. 12 , pp. 3025-3035, 2008.

[5] T. H. Summers, M. R. Akella, and M. J. Mears, "Coordinated standoff tracking of moving targets: Control laws and information architectures," Journal of Guidance, Control, and Dynamics, vol. 32, no. 2, pp. 56-69, 2009.

[6] I. Shames, B. Fidan, and B. D. O. Anderson, "Close target reconnaissance with guaranteed collision avoidance," International Journal of Nonlinear and Robust Control, vol. 21, no. 16, p. 18231840, 2011.

[7] H. Khalil, Nonlinear Systems. Prentice-Hall, 2002.

[8] Z. Qu, Cooperative control of dynamical systems. Springer, 2009.

[9] B. D. O. Anderson, "Exponential stability of linear equations arising in adaptive identification," IEEE Transactions on Automatic Control, vol. 22, no. 1, pp. 83-88, 1977.

[10] S. Sastry and M. Bodson, Adaptive Control: Stability, Convergence, and Robustness. Prentice-Hall, 1994.

\footnotetext{
5 If $\rho(0) \rightarrow 0$, then for $\gamma \in(0, \pi), \dot{\gamma}(t) \rightarrow+\infty$ and for $\gamma \in(\pi, 2 \pi)$, $\dot{\gamma}(t) \rightarrow-\infty$. So, for $\rho(0) \rightarrow 0, \gamma(t)$ goes to $\pi$.
} 